\title{
REMARKS ON THE TWO WELL PROBLEM WITH ROTATIONS
}

\author{
KEWEI ZHANG
}

(Communicated by Barbara L. Keyfitz)

\begin{abstract}
A conjecture of I. Fonseca on the form of minimizers of a doublewell problem arising in the study of elastic crystal microstructure is answered to be true.
\end{abstract}

In the study of continuum models for phase transitions in elastic solids, Fonseca [1] employs a penalization method to a double-well model with rotations, showing that a sequence of minimizers of the approximation problem admits a subsequence converging weakly to a solution of the unperturbed problem of the form

$$
\nabla u(x)=R(x)\left(B+\chi_{E}(x) \mathbf{a} \otimes \mathbf{n}\right) \quad \text { in } \Omega \subset \mathbb{R}^{3}
$$

\section{subject to}

(2) $\int_{\Omega} \nabla u d x=\operatorname{meas}(\Omega)(B+\theta \mathbf{a} \otimes \mathbf{n}), \quad 0<\theta<1, \quad \int_{\Omega} u d x=m$,

where $R(x) \in \mathrm{SO}(3)$ is a rotation, $B$ is a $3 \times 3$ matrix with $\operatorname{det} B>0, \nabla u, R$ and $\chi_{E} \in B V(\Omega) \cap L^{\infty}(\Omega), u \in W^{1, \infty}\left(\Omega ; \mathbb{R}^{3}\right), \nabla u$ is the gradient of $u$, and $\chi_{E}$ is the characteristic function of some measurable set $E \subset \Omega$. The main conjecture in [1] is that $R=I$-the unit matrix. The conjecture is confirmed if the set $E$ determines a partition of $\Omega$ into countably many open, strongly Lipschitz connected domains [1, Theorem 5.8], or a is parallel to $B^{-\mathrm{T}} \mathbf{n}$. In this note we prove that Fonseca's conjecture is true, that is,

Theorem 1. Let $\Omega \subset \mathbb{R}^{3}$ be open and bounded; suppose that $u \in W^{1, \infty}\left(\Omega, \mathbb{R}^{3}\right)$ is such that

$$
\begin{array}{rlrl}
\nabla u(x) & =R(x)\left(B+\chi_{E}(x) \mathbf{a} \otimes \mathbf{n}\right) & & \text { in } \Omega, \\
\int_{\Omega} \nabla u d x=\operatorname{meas}(\Omega)[B+\theta \mathbf{a} \otimes \mathbf{n}], & & 0<\theta<1,
\end{array}
$$

where $R \in \mathrm{SO}(3)$ a.e.; $\operatorname{det} B \neq 0, \mathbf{a}, \mathbf{n} \in \mathbb{R}^{3}, \mathbf{a} \neq 0,|\mathbf{n}|=1 ; E \subset \Omega$ is measurable. Then $R=I$ a.e.

Proof. Since $\nabla u(x)=R(x)\left(I+\chi_{E}(x) \mathbf{a} \otimes\left(B^{-\mathrm{T}} \mathbf{n}\right)\right) B$, by a change of coordinates we may assume that

$$
\nabla u=R^{\prime}(x)\left(I+\chi_{E} \mathbf{a}^{\prime} \otimes \mathbf{n}^{\prime}\right), \quad \int_{\Omega} \nabla u d x=\operatorname{meas}(\Omega)\left[I+\theta \mathbf{a}^{\prime} \otimes \mathbf{n}\right]
$$

Received by the editors April 22, 1991 and, in revised form, June 19, 1991.

1991 Mathematics Subject Classification. Primary 49B36, 49A50, 73C50. 
where $R^{\prime}(x)=R\left(B^{-1} x\right)$, and we may find some $Q \in \mathrm{SO}(3)$ such that

$$
\begin{gathered}
Q \mathbf{n}^{\prime}=\mathbf{e}_{1}, \quad Q \mathbf{a}^{\prime}=\alpha \mathbf{e}_{1}+\beta \mathbf{e}_{2}, \quad \mathbf{e}_{1}=(1,0,0)^{\mathrm{T}}, \quad \mathbf{e}_{2}=(0,1,0)^{\mathrm{T}}, \\
Q \nabla u Q^{\mathrm{T}}=Q R(x) Q^{\mathrm{T}}\left[Q I Q^{\mathrm{T}}+\chi_{E} Q \mathbf{a}^{\prime} \otimes\left(Q^{\mathrm{T}} \mathbf{n}^{\prime}\right)\right] .
\end{gathered}
$$

Hence by a further change of coordinates, we may assume that

$$
\begin{gathered}
\nabla u=R(x)\left[I+\chi_{E} \alpha e_{1} \otimes e_{1}+\beta e_{2} \otimes e_{1}\right], \quad \beta \neq 0, \\
\int_{\Omega} \nabla u d x=\operatorname{meas}(\Omega)\left[I+Q\left(\alpha e_{1} \otimes e_{1}+\beta e_{2} \otimes e_{1}\right)\right] .
\end{gathered}
$$

We have

$$
\nabla u=\left(\nabla u_{1}, \nabla u_{2}, \nabla u_{3}\right)^{\mathrm{T}}, \quad R=\left(R_{1}, R_{2}, R_{3}\right)^{\mathrm{T}}, \quad R_{i}=\left(R_{i 1}, R_{i 2}, R_{i 3}\right)^{\mathrm{T}},
$$

and so

$$
\int_{\Omega} \nabla u_{3} d x=\int_{\Omega} R_{3} d x=\operatorname{meas}(\Omega)(0,0,1)^{\mathrm{T}} .
$$

Since $\left|R_{3}\right|=1$, a.e. we deduce that $R_{33}=1$ a.e. $R_{31}=R_{32}=0$ a.e.

Since $R \in \mathrm{SO}(3)$, we have

$$
R(x)=\left(\begin{array}{ccc}
R_{11}(x) & R_{12}(x) & 0 \\
R_{21}(x) & R_{22}(x) & 0 \\
0 & 0 & 1
\end{array}\right) \quad \text { and } \quad\left(\begin{array}{ll}
R_{11} & R_{12} \\
R_{21} & R_{22}
\end{array}\right) \in \mathrm{SO}(2)
$$

a.e.

From (3), we have $\partial u_{2} / \partial x_{2}=R_{22}(x)$, with $\left|R_{22}(x)\right| \leq 1$ a.e., and

$$
\int_{\Omega} \frac{\partial u_{2}}{\partial x_{2}} d x=\operatorname{meas}(\Omega)\left[I+\theta\left(\alpha e_{1} \otimes e_{1}+\beta e_{2} \otimes e_{1}\right)\right]_{22}=\operatorname{meas}(\Omega),
$$

which implies $R_{22}=1$ a.e. Therefore (4) implies $R_{11}=1, R_{12}=R_{21}=0$.

Remark 1. Since $R=I$, we have $\nabla u=B+\chi_{E} \mathbf{a} \otimes \mathbf{n}$, and so

$$
\text { curl } \nabla u=0 \text { in the sense of distributions, }
$$

which holds if and only if the outward normal $v$ to $\partial E \cap \Omega$ is parallel to $\mathbf{n}$.

Remark 2. The proof of the conjecture depends heavily on the constraints (2), which seems to be a very strong condition to ensure that $R(x)=$ Identity. I do not know what happens without this constraint. I conjecture that $R(x)=$ $R_{0} \in \mathrm{SO}(3)$ is a constant matrix in $\mathrm{SO}(3)$ without assuming (2). (Also see [1, Corollary 5.20].)

\section{ACKNOWLEDGMENT}

I am thankful to the referee for valuable suggestions.

\section{REFERENCES}

1. I. Fonseca, Phase transitions of elastic solid materials, Arch. Rational Mech. Anal. 107 (1989), 195-223.

Current address: School of Mathematics, Physics, Computing and Electronics, Macquarie University, North Ryde, New South Wales 2109, Australia

E-mail address: kewei@macadam.mpce.mq.edu.au 